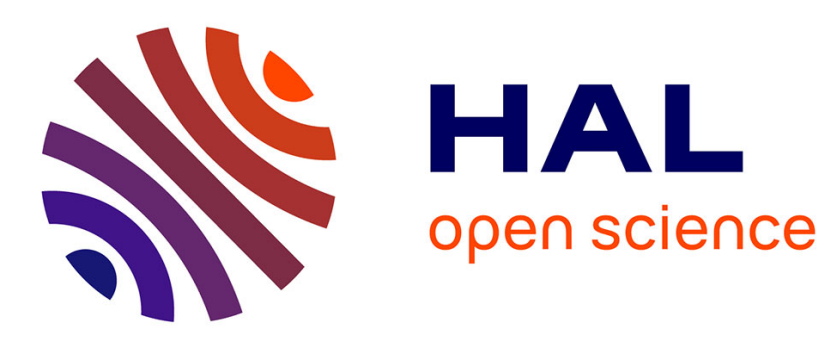

\title{
Anomalous diffusion mediated by atom deposition into a porous substrate.
}

Pascal Brault, Christophe Josserand, Jean-Marc Bauchire, Amaël Caillard, Christine Charles, Rod W. Boswell

\section{- To cite this version:}

Pascal Brault, Christophe Josserand, Jean-Marc Bauchire, Amaël Caillard, Christine Charles, et al.. Anomalous diffusion mediated by atom deposition into a porous substrate.. Physical Review Letters, 2009, 102, pp.045901. 10.1103/PhysRevLett.102.045901 . hal-00355521

\section{HAL Id: hal-00355521 \\ https://hal.science/hal-00355521}

Submitted on 22 Jan 2009

HAL is a multi-disciplinary open access archive for the deposit and dissemination of scientific research documents, whether they are published or not. The documents may come from teaching and research institutions in France or abroad, or from public or private research centers.
L'archive ouverte pluridisciplinaire HAL, est destinée au dépôt et à la diffusion de documents scientifiques de niveau recherche, publiés ou non, émanant des établissements d'enseignement et de recherche français ou étrangers, des laboratoires publics ou privés. 


\title{
Anomalous diffusion mediated by atom deposition into a porous substrate.
}

\author{
Pascal Brault,, , $\rightarrow$ Christophe Josserand, ${ }^{2,3}$ Jean-Marc Bauchire, ${ }^{1}$ \\ Amaël Caillard, ${ }^{1,4}$ Christine Charles, ${ }^{4}$ and Rod W. Boswell ${ }^{4}$ \\ ${ }^{1}$ Groupe de Recherches sur l'Energétique des Milieux Ionisés, \\ UMR6606 CNRS-Université d'Orléans BP 6744, 45067 Orléans Cedex 2, France \\ ${ }^{2}$ Institut Jean Le Rond d'Alembert, UMR 7190 CNRS-Paris VI Case 162, \\ UPMC, 4 place Jussieu, 75252 Paris Cedex 05, France \\ ${ }^{3}$ Kavli Institute for Theoretical Physics, University of California, Santa Barbara, CA 93106,USA \\ ${ }^{4}$ Space Plasma, Power, and Propulsion group (SP3), \\ Research School of Physical Sciences and Engineering, \\ The Australian National University, Canberra, ACT 0200, Australia
}

\begin{abstract}
Constant flux atom deposition into a porous medium is shown to generate a dense overlayer and a diffusion profile. Scaling analysis shows that the overlayer acts as a dynamic control for atomic diffusion in the porous substrate. This is modeled by generalizing the porous diffusion equation with a time-dependent diffusion coefficient equivalent to a nonlinear rescaling of time.
\end{abstract}

PACS numbers: 66.30.Pa, 66.30.Dn, 68.55.-a, 81.15.Cd

Thin film depositions on substrates are important in many physical processes and applications. Moreover, deposition on porous substrates is particularly useful for catalytic systems [1, 2] in which atomic deposition carried out by plasma sputtering is then coupled with the diffusion of the atoms into the porous substrate. Diffusion in a porous medium is generally anomalous and is characterized by the mean square displacement $\left\langle z^{2}(t)\right\rangle$ : it evolves with a power law in time $t^{\alpha}$ different from the well known linear behavior $(\alpha=1)$ for normal diffusion. Anomalous diffusion [3, 4] is a general process that can be observed in many domains such as transport in porous [5] and/or fractal media [6], surface growth [7], solid surface diffusion 8] or hydrodynamics (rotating flows [9], turbulence [10] or diffusion in an array of convection rolls [11, 12]). It can be described by different models that involve space-dependent diffusion coefficients [3, 4, 13, 14, 15, 16, 17]. In these models anomalous diffusion is included through the propagator (the solution of the diffusion equation starting with a Dirac distribution at $t=0$ ) that exhibits both power laws and stretched exponential behaviors. Here, we study platinum deposition by plasma sputtering on a porous carbon substrate. The platinum atoms are sputtered by the plasma ions and travel to the substrate so that both deposition and diffusion processes can depend on plasma operating parameters. The experimental results consist in a measure of the density profiles of the deposited matter at different times. The goal of this Letter is to characterize this diffusion-deposition process in a porous carbon medium using the time evolution of the platinum density profile.

Platinum atoms are deposited by plasma sputtering into a porous carbon layer supported (See Fig. 11) on

\footnotetext{
*corresponding author Pascal.Brault@univ-orleans.fr
}

a carbon cloth. This porous layer is a few tens of microns thick and is composed of randomly stacked carbon nanoparticles (Vulcan XC 72) and PTFE particles brushed onto the carbon cloth. The specific area is $15 \mathrm{~m}^{2} \mathrm{~g}^{-1}$ before $\mathrm{Pt}$ deposition and slightly lower at 13 $\mathrm{m}^{2} \mathrm{~g}^{-1}$ after Pt deposition. Examination of Fig 1 shows the pore size reduction. Platinum atoms are deposited onto this porous carbon layer and diffuse in the course of deposition. The platinum deposition reactor is a previously described [1, 2] plasma sputter system which delivers a constant deposition rate. An argon plasma is created in the stainless steel deposition chamber $18 \mathrm{~cm}$ inner-diameter and $25 \mathrm{~cm}$-long by using an external planar antenna (also known as TCP antenna). The porous layer is placed on a movable grounded substrate holder in front of the platinum sputter target with a targetsubstrate distance of $4.5 \mathrm{~cm}$. The porous layer is thus exposed to a flux of sputtered platinum, into which it diffuses under the operating conditions. The diffusion process was studied at argon pressures of 0.5 and $5 \mathrm{~Pa}$, leading to a mean kinetic energy $E_{k}$ of the sputtered Pt atom around $7 \mathrm{eV}$ [2] and $0.04 \mathrm{eV}$ respectively [18]. The resulting platinum depth profiles were measured using Rutherford Backscattering Spectroscopy, which gives an indirect measure of the average density as a function of the depth $z$. The density is obtained by fitting the experimental spectrum with a spectrum derived from a defined profile function. Firstly, we observe that the platinum profile can in fact be decomposed in two distinct regions: a growing layer above the porous medium, of mean thickness $h(t)=z_{0}(t)$ and constant density $\rho(z, t)=Z_{1}(t),-z_{0}(t)<z \leq 0$ and a density profile $\rho(z, t), z>0$ in the porous medium (which extends in the $z>0$ domain, $z=0$ corresponding to the porous interface). The $\mathrm{Pt}$ depth profile is thus deduced by minimizing the difference between the experimental RBS spectrum and the simulated RBS spectrum using the profile de- 

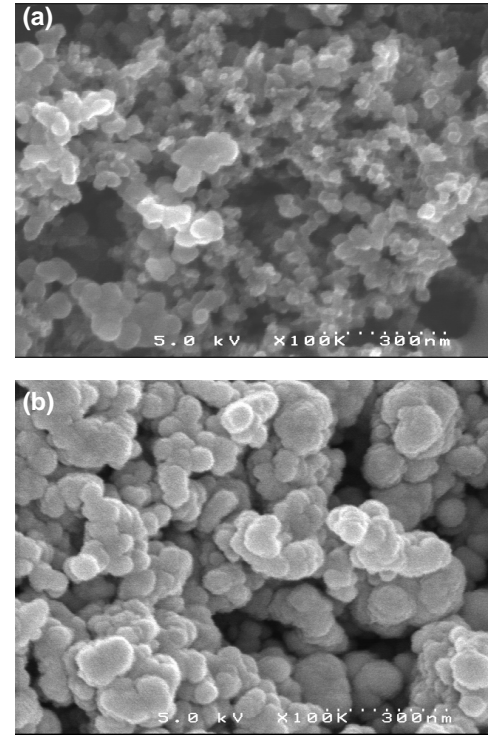

FIG. 1: Scanning Electron Microscopy top view of porous carbon layer a) before $\mathrm{Pt}$ deposition b) after Pt deposition (Courtesy D. Cot, IEMM CNRS-ENSCM-Univ. Montpellier II)

fined by Eq. 1. It corresponds to the known solution of anomalous diffusion processes [2] i.e. a stretched exponential.

$$
\begin{array}{rc}
\rho(z, t)=Z_{1}(t), & -z_{0}<z \leq 0 \\
\rho(z, t)=Z_{1}(t) e^{-\frac{z^{2}+\theta}{Z_{2}(t)}}, & z>0
\end{array}
$$

where $\theta$ is the dimensionless coefficient which characterizes the anomalous diffusion behavior 13, 14, 15].This fitting procedure leads to the error bar of the exponent $\theta$ as reported hereafter. The density profile is continuous at the interface between the growing platinum layer and the porous medium at $z=0$ so that we have $\rho(0, t)=Z_{1}(t)$. $Z_{2}(t)$ is the time dependent spreading of the stretched exponential. Such a profile suggests that the particle flux delivered by the plasma sputtering cannot be absorbed directly by the porous layer with the result that a fraction of the incident atoms have to deposit at the interface. It also implies that the diffusion process into the porous layer is a consequence of both the diffusion of the platinum atoms that have penetrated into the substrate and the diffusion of those absorbed at the interface.

Some fitted platinum depth profiles at different deposition times $t$ in the porous medium are displayed in Fig. 2. The best fits correspond to $\theta=-1.45 \pm 0.05 \approx-3 / 2$ which suggests a super-diffusive behavior in the porous medium (see below). Moreover, given $\theta=-1.5, Z_{1}(t)$ and $Z_{2}(t)$ asymptotically follow power law behaviors with time $\left(Z_{1} \sim t^{m}\right.$ and $\left.Z_{2} \sim t^{p}\right)$ with $m$ being $0.40 \pm 0.05$ and $p$ being $0.20 \pm 0.05$ as shown in Fig. 3(a). $h(t)$ also obeys a less well defined power law behavior with

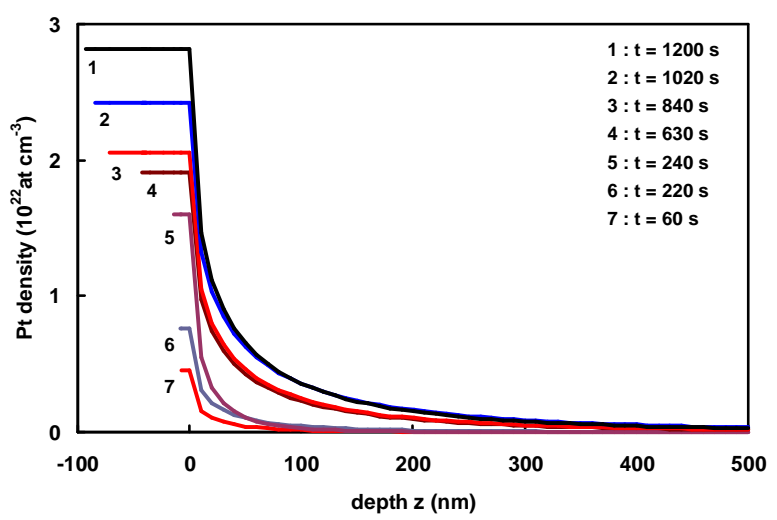

FIG. 2: (Color online) Pt depth profiles in porous carbon at different successive deposition times. $z=0$ corresponds to the porous carbon layer surface

$\sim t^{n}, n=1.1 \pm 0.1$. The mass of platinum inside the porous medium is found to scale as $t^{0.8 \pm 0.1}$ while the $\mathrm{Pt}$ mass of the surface overlayer, $Z_{1}(t) \cdot h(t)$, scales as $t^{1.5 \pm 0.1}$ as shown on Fig. 3(b). However, we obtain an almost linear evolution of the total mass $\left(\sim t^{1.1 \pm 0.1}\right)$ of platinum deposited in and on the porous medium, as expected by the experimental process. At the higher pressure, $5 \mathrm{~Pa}$, $\theta=-1.33 \pm 0.05, m=p=0.25 \pm 0.05$ and $n=1.0 \pm 0.1$. Hence, a change of deposition conditions yields a change of $\mathrm{Pt}$ concentration profile within the porous carbon. To help explain our results, we use a classical model of the anomalous diffusion process [13, 14, 15, 16] in fractal and porous media, considered here in one dimensional space accounting for the in plane averaging (we can discard nonlinear diffusion in the porous media from the experimental results) resulting in the following generalized diffusion equation for the density profile $\rho(z, t)$ for $z \geq 0$ :

$$
\frac{\partial \rho}{\partial t}=\frac{\partial}{\partial z}\left(\frac{K_{0}}{z^{\theta}} \frac{\partial \rho}{\partial z}\right)
$$

where $\theta$ is a real parameter characterizing anomalous diffusion and is a priori unknown. This equation is obtained by postulating a distance dependence $\frac{K_{0}}{z^{\theta}}$ of the diffusion coefficient in Fick's law. Classical Brownian diffusion holds for $\theta=0$, whereas positive (negative) $\theta$ corresponds to sub(super)-diffusive dynamics where the mean-square displacement scales as $<z^{2}>\propto t^{2 /(2+\theta)}$. The general solution for this equation (the so-called propagator) exhibits a power law and stretched exponential behavior and is given by [4, 13, 14, 15, 16, 17]:

$$
\rho(z, t) \propto\left[K_{0}(2+\theta)^{2} t\right]^{-1 /(2+\theta)} \exp \left[-\frac{z^{2+\theta}}{K_{0}(2+\theta)^{2} t}\right]
$$

which holds for a normalization condition consistent with mass conservation. Since the deposition is carried out with a constant flux, the total mass is expected a priori 

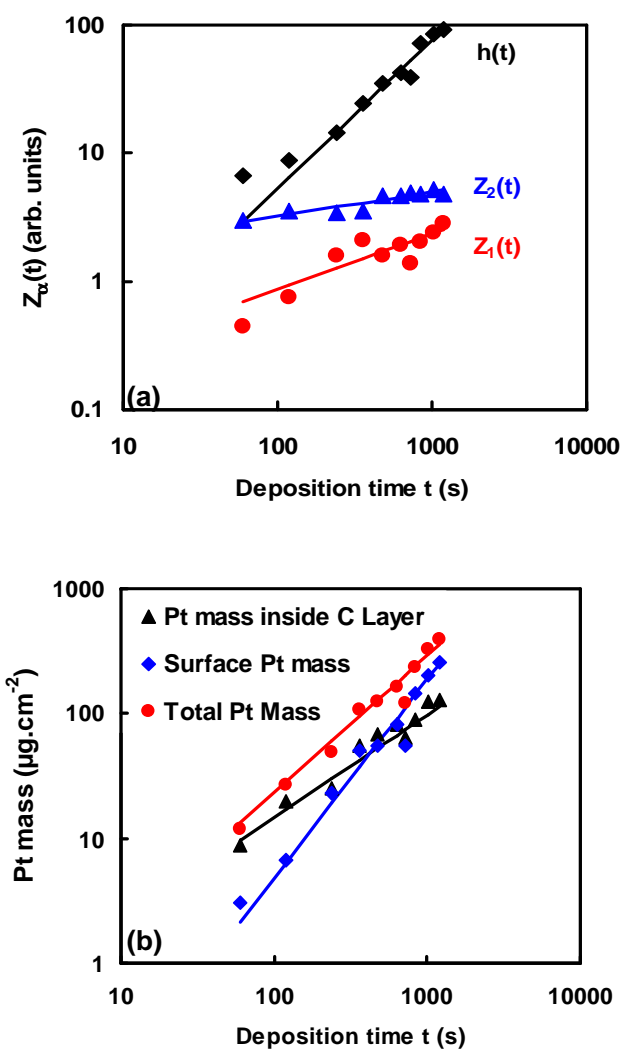

FIG. 3: (Color online) For the 0.5 Pa deposition, a) thickness of the Pt layer $h(t)$ above the porous medium and value of $Z_{1}(t)$ and $Z_{2}(t)$ from the fitted law (1) as a function of time (log-log plot). The lines correspond to the power laws $Z_{1}(t) \sim$ $t^{0.4}, Z_{2}(t) \sim t^{0.2}$ and $h(t) \sim t^{1.1}$; b) Amount of Pt mass in the porous medium calculated using (11) as a function of time (log$\log$ plot).

to grow linearly and the solution to our problem should obey the boundary condition at $z=0$ :

$$
\frac{K_{0}}{z^{\theta}} \frac{\partial \rho}{\partial z}=C_{0}
$$

where $C_{0}$ is the constant flux of platinum imposed by the plasma sputtering. Therefore, the solution can be obtained using the propagator solution (3) through a linear superposition consistent with the boundary condition (44). It is in fact more convenient to investigate the solution in the following self-similar form:

$$
\rho(z, t)=t^{\beta} f\left(\frac{z}{t^{\alpha}}\right) .
$$

While the propagator obeys $\alpha+\beta=0$ (constant mass solution), the constant flux solution must have $\alpha+\beta=1$. Inserting (5) into the diffusion equation (2) we obtain $\alpha=\frac{1}{2+\theta}$ while $\beta$ is fixed by the flux condition. The self-similar function $f(\xi)$ is then the solution of the ordinary differential equation $\left(\xi=z / t^{\alpha}\right.$ being the self-similar variable):

$$
\beta f-\alpha \xi f^{\prime}=\frac{K_{0}}{\xi^{\theta+1}}\left(\xi f^{\prime \prime}-\theta f^{\prime}\right)
$$

which gives the propagator solution (3) for $\alpha+\beta=0$. Moreover, it can be seen that the density profile for large $z$ follows the stretched exponential law (1) with $Z_{1}(t) \propto t^{\beta}$ and $Z_{2}(t)=K_{0}(2+\theta)^{2} t$.

The predictions of this simple model do not agree with the experimental results: from $\theta=-1.5$, one would obtain $\alpha=2$ and $\beta=-1$ since we have the condition $\alpha+\beta=1$. Hence, there is a marked discrepancy between the experimental measurements $(\alpha \sim 0.4$ and $\beta \sim 0.4)$ and the model even when the error bars of the experiments are taken into account. However, the underlying physical processes of atomic diffusion and the measured density profiles suggest that the general diffusion equation proposed here is a good framework for modeling atomic deposition through plasma sputtering. It is thus tempting to model the effect of the growing external platinum layer and of the plasma by keeping the same equation with the anomalous diffusion coefficient $K(t)$ and a flux of mass at $z=0$, both time dependent. Although we are far from a detailed microscopic derivation of the model, it is postulated here that the growth of the platinum layer has a screening effect on the diffusion and thus alters the mass flux towards the porous medium. In particular the platinum overlayer acts as a reservoir for diffusion. In addition, the temperature distribution, not accounted for directly by this model, is certainly influenced by the presence of the platinum layer. Since the diffusion coefficient is a function of the temperature, it is probably time dependent. This time dependent diffusion coefficient can also be understood as a time rescaling and it is needed in the framework of the diffusive equation since it is the only way to change the time dependence in the exponential law (31). We thus propose to model the deposition/diffusion dynamics through the previous equation (2) with a time dependent diffusion coefficient $K(t)$ :

$$
\frac{\partial \rho}{\partial t}=\frac{\partial}{\partial z}\left(\frac{K(t)}{z^{\theta}} \frac{\partial \rho}{\partial z}\right)
$$

The presence of the external growing layer is also accounted for by an a priori time dependent boundary condition:

$$
\frac{K(t)}{z^{\theta}} \frac{\partial \rho}{\partial z}=C(t)
$$

To illustrate the results with no loss of generality, we will seek a power law behavior for a sufficiently time. We introduce two additional exponents $\epsilon$ and $\gamma$ such that the flux of atoms and the diffusion coefficient follow:

$$
C(t)=C_{0} t^{\epsilon} \quad K(t)=K_{0} t^{\gamma}
$$

The relations between the exponents are straightforward:

$$
\alpha=\frac{\gamma+1}{2+\theta} \quad \text { and } \quad \alpha+\beta=1+\epsilon
$$


Eq. (7) can be understood by rescaling the time following $\tau=t^{1+\gamma}$ in terms of the initial diffusion equation (2):

$$
\frac{\partial \rho}{\partial \tau}=\frac{\partial}{\partial z}\left(\frac{K_{0}}{1+\gamma} \frac{1}{z^{\theta}} \frac{\partial \rho}{\partial z}\right)
$$

And we obtain the stretched exponential behavior for large $z$ in agreement with the experimental measurements:

$$
\rho(z, t) \propto t^{\beta} \exp \left[-\frac{(1+\gamma) z^{2+\theta}}{K_{0}(2+\theta)^{2} t^{1+\gamma}}\right]
$$

Within this general approach, we can now describe our experimental results. For simplicity's sake and to illustrate the model with no loss of generality, we estimate from the experimental fits that $\theta=-\frac{3}{2}, \alpha=0.4$ and $\beta=0.4$ which leads to:

$$
\gamma=-0.8 \quad \text { and } \quad \epsilon=-0.2
$$

This corresponds to a time decreasing diffusion coefficient such as $t^{-0.8}$ and of the flux at $z=0$ such as $t^{-0.2}$. The diffusion coefficient is expected to decrease versus time, while the porous medium is gradually filled by the platinum atoms. This leads to a more difficult diffusion. At the $z=0$ interface, the growing external layer provides an additional source for the diffusion of atoms inside the porous substrate. Moreover a decreasing flux indicates that the growing external layer is gradually screening the influence of the plasma, and that diffusion becomes less efficient due to pore filling as mentioned above. These effects are also observed at $5 \mathrm{~Pa}$, with $\theta=-\frac{4}{3}, \alpha=0.5$ and $\beta=0.25$ which leads to: $\gamma=-0.83$ and $\epsilon=-0.25$. These last two scaling exponents are very close to those at $0.5 \mathrm{~Pa}$. Consequently, the self similar exponents depend on the deposition conditions whereas the critical exponents of the diffusion are unchanged. This means that the diffusion equations (17]8,[11) are robust enough to provide a general frame for deposition/diffusion mechanisms.

On the basis of experimental results and a general diffusion model, we have proposed a new scenario for mass deposition and diffusion on a porous substrate. As the flux of sputtered atoms cannot be absorbed immediately by the substrate, an overlayer grows. The combined effects of the deposition that change the porosity properties of the substrate and of this growing layer that can act as a reservoir for diffusion can be accounted for by generalizing a standard model of diffusion in a porous medium with constant diffusive properties ( $\theta$ is constant). This allows time dependent coefficients to be introduced. This model provides a clear understanding of the experimental results: in particular, we show that the diffusion coefficient in the porous substrate and the flux at the interface decrease with time due to the atom deposition in the substrate. This general model can be also be applied to many other diffusion processes where the inner porous structure of a material is modified by a penetrating flux of matter, as for example, for filtration through porous membranes, sedimentation processes, ...

The CNRS is acknowledged for a Ph.D. scolarship (A. Caillard). Université d'Orléans (BQR 2002) and the Région Centre is acknowledged for funding of the experimental setup. C.J. thanks the DGA for financial support. M. Mikikian is acknowledged for a careful reading of the manuscript. The reviewers are gratefully acknowledged for useful comments.
[1] P. Brault, A. Caillard, A. L. Thomann, J. Mathias, C. Charles, R. W. Boswell, S. Escribano, J. Durand and T. Sauvage, J. Phys. D 34, 3419 (2004).

[2] A. Caillard, P. Brault, J. Mathias, C. Charles, R. W. Boswell and T. Sauvage, Surf. Coat. Technol. 200, 391 (2005).

[3] J.-P. Bouchaud and A. Georges, Phys. Rep. 195, 127 (1990).

[4] R. Metzler and J. Klafter, Phys. Rep. 3391 (2000).

[5] D. G. Aronson The Porous Media Equation. In: Nonlinear Diffusion Problems (A. Fasano and M. Primicerio Eds., Springer-Verlag, Berlin, 1986)

[6] J. Stephenson, Physica A 222, 234 (1995).

[7] H. Spohn, J. Phys. I France 3, 69 (1993).

[8] W.D. Luedtke and U. Landman, Phys. Rev. Lett. 82, 3835 (1999).

[9] E.R. Weeks and H.L. Swinney, Phys. Rev. E 57, 4915 (1998).
[10] L.F. Richardson, Proc. Roy. Soc. 110, 709 (1926).

[11] O. Cardoso and P. Tabeling, Europhys. Lett. 7, 225 (1988).

[12] W. Young, A. Pumir and Y. Pomeau, Phys. Fluids A $\mathbf{1}$, 462 (1989).

[13] L. C. Malacarne, R. S. Mendes, I. T. Pedron and E. K. Lenzi, Phys. Rev. E 63, 030101(R) (2001).

[14] I. T. Pedron, R. S. Mendes, L.C. Malacarne and E. K. Lenzi, Phys. Rev. E 65, 041108 (2002).

[15] C. Tsallis and E. K. Lenzi, Chem. Phys. 284, 341 (2002).

[16] B. O'Shaughnessy and I. Procaccia Phys. Rev. Lett. 54, 455 (1985).

[17] B. O'Shaughnessy and I. Procaccia, Phys. Rev. A 32, 3073 (1985).

[18] A. Caillard, C. Charles, R. Boswell, A. Meige, P. Brault, Plasma Sources Sci. Technol. 17, 035028 (2008) 